\title{
How much information is lost by using global-mean climate metrics? an example using the transport sector
}

\author{
M. T. Lund • T. Berntsen • J. S. Fuglestvedt • \\ M. Ponater $\cdot$ K. P. Shine
}

Received: 29 July 2011 / Accepted: 12 December 2011 /Published online: 11 January 2012

(C) Springer Science+Business Media B.V. 2012

\begin{abstract}
Metrics are often used to compare the climate impacts of emissions from various sources, sectors or nations. These are usually based on global-mean input, and so there is the potential that important information on smaller scales is lost. Assuming a non-linear dependence of the climate impact on local surface temperature change, we explore the loss of information about regional variability that results from using global-mean input in the specific case of heterogeneous changes in ozone, methane and aerosol concentrations resulting from emissions from road traffic, aviation and shipping. Results from equilibrium simulations with two general circulation models are used. An alternative metric for capturing the regional climate impacts is investigated. We find that the application of a metric that is first calculated locally and then averaged globally captures a more complete and informative signal of climate impact than one that uses global-mean input. The loss of information when heterogeneity is ignored is largest in the case of aviation. Further investigation of the spatial distribution of temperature change indicates that although the pattern of temperature response does not closely match the pattern of the forcing, the forcing pattern still influences the response pattern on a hemispheric scale. When the short-lived transport forcing is superimposed on present-day anthropogenic $\mathrm{CO}_{2}$ forcing, the heterogeneity in the temperature response to $\mathrm{CO}_{2}$ dominates. This suggests that the importance of including regional
\end{abstract}

Electronic supplementary material The online version of this article (doi:10.1007/s10584-011-0391-3) contains supplementary material, which is available to authorized users.

M. T. Lund $(\bowtie) \cdot$ T. Berntsen $\cdot$ J. S. Fuglestvedt

CICERO - Center for International Climate and Environmental Research, Oslo, Norway

e-mail: m.t.lund@cicero.uio.no

T. Berntsen

Department of Geosciences, University of Oslo, Oslo, Norway

M. Ponater

Deutsches Zentrum für Luft- und Raumfahrt, Institut für Physik der Atmosphäre, Oberpfaffenhofen, Germany

K. P. Shine

Department of Meteorology, University of Reading, Reading, UK 
climate impacts in global metrics depends on whether small sectors are considered in isolation or as part of the overall climate change.

\section{Introduction}

Global-mean metrics are commonly used for quantifying the impact of human activity on climate (e.g. Forster et al. 2007; IPCC 2009; Fuglestvedt et al. 2010). Metrics, such as the Global Warming Potential (GWP), are commonly used as a tool for aggregating information and for placing emissions of different gases on a common scale. They are particularly useful when comparing and evaluating the climate effects of several emission components, sources or sectors (Rive et al. 2007; Berntsen and Fuglestvedt 2008; Fuglestvedt et al. 2008; Unger et al. 2008, 2009; Skeie et al. 2009) and they are frequently applied to assess the global consequences of possible mitigation measures.

Such metrics normally use globally-average input to produce globally-average measures and give no information about the spatial variability of the impact. Even for relatively homogeneous changes in greenhouse gas concentrations (such as carbon dioxide and methane) the climate response (including that of surface temperature) is quite inhomogeneous (e.g. Boer and $\mathrm{Yu}$ 2003). Many other perturbations of atmospheric species, especially short-lived ones, produce a distinctly heterogeneous radiative forcing which causes further inhomogeneity in the response which can be strongly dependent on the location of the forcing (Forster et al. 2000; Joshi et al. 2003; Berntsen et al. 2005; Shindell et al. 2010). Additionally the sign of the forcing (and hence response) varies between different species. Moving further down the cause and effect chain, climate change leads to social and ecological impacts. These are likely to depend on the regional distribution of the climate change and may also have a non-linear dependence on that change (Kandlikar 1995, 1996; Manne et al. 1995; Hammitt et al. 1996; Tol 2002). In the case of such spatial variability in the magnitude and sign of the response and impacts, global averaging may lead to cancellations so that the strength of regional impacts is hidden. This problem has been pointed out in several studies (e.g. Fuglestvedt et al. 2003; Tol et al. 2004; Shine et al. 2005; Shindell and Faluvegi 2010; Joshi et al. 2011).

In this paper we investigate one method for including information on spatially variable climate impacts in global metrics and explore how the loss of information about impact by global averaging can influence the strength and ranking of the global climate impacts of the transport sector. The problems related to the use of global-mean indicators or metrics in the case of spatially heterogeneous perturbations are well-known and our study takes a step towards actually quantifying how significant the loss of information can be in specific cases with a focus on application for development of metrics. Using results from simulations with two General Circulation Models (GCMs), we first investigate the heterogeneity in the net surface air temperature response to ozone $\left(\mathrm{O}_{3}\right)$ and methane $\left(\mathrm{CH}_{4}\right)$ perturbations (i.e. the net effect of emissions of ozone precursors $\mathrm{NOx}, \mathrm{CO}$ and VOC) from three transport sectors: road traffic, aviation and shipping. We then expand the analysis to include perturbations of aerosols for each sector. Our study systematically compares the heterogeneity in response, and the following loss of information by global averaging, across models, sectors and components.

The decision to focus on ozone precursors is motivated by the fact that these are important components in the emissions from the transport sectors, with the majority of emissions occurring in the Northern hemisphere. However, the net climate impact via changes in atmospheric $\mathrm{O}_{3}$ and $\mathrm{CH}_{4}$ is difficult to determine, particularly for $\mathrm{NOx}$, due to inhomogeneities in forcing and response and due to compensating effects. Some of the difficulties related to NOx are discussed in e.g. Fuglestvedt et al. $(1999,2010)$ and Shine et al. (2005). The NOx-induced increase in $\mathrm{O}_{3}$ and the resulting climate response depends on 
the location of emissions. NOx emissions also lead to a longer-lived, more homogeneous cooling effect due to reductions in $\mathrm{CH}_{4}$; climate model simulations presented in Shine et al. (2005) indicate that the net effect of Northern hemisphere NOx emissions is a warming in this hemisphere, but a cooling in the Southern hemisphere. While these opposing effects can be of nearly the same order globally, and hence largely cancel, the regionality in the response means that the regional or local impact may not cancel.

The relationship between climate change and the impact it causes is very uncertain. Impact, or damage, functions can be defined in numerous ways and for different impact categories, such as agriculture, health or ecosystems (Nordhaus and Boyer 2000; Tol 2002). In economic studies impact is normally valued in monetary terms, often using a discounting rate for weighting future impacts. The impact caused by a certain change in e.g. temperature can also have significant regional difference and the impact functions can be defined as a function of the regional change (Nordhaus and Boyer 2000). Furthermore, impacts can be weighted, e.g. according to population or agricultural activity. One could for instance argue that a temperature increase causes the most serious impact in populated regions or that impact functions for agriculture should be weighted by agricultural area or production.

Estimating the impact of climate change requires assumptions about whether this impact has linear, quadratic or some other functional dependence on the change. We assume non-linear impact in this study and the impact is taken to be the square of the temperature change, a relationship which has been used in several previous studies (Kandlikar 1995, 1996; Manne et al. 1995; Hammitt et al. 1996); however an exact form has not been established. We build on the main idea from Shine et al. (2005) and use two different simplified indicators, or metrics, to calculate global climate impact of ozone precursor and aerosol emissions from transport. Our study moves one step forward by calculating the climate impact of a specific sector and by including a larger set of components. For the purpose of illustration we first apply a metric assuming that the impact is given as the global-mean temperature change squared, i.e. using global-mean input. The impact may also be represented by a metric that includes more of the spatial heterogeneity in temperature change. The results are therefore compared to an alternative metric which is first calculated locally and then averaged globally in order to see if we can capture a more complete and informative signal, i.e. more representative of the true impact as this alternative metric accounts for aspects of regional variability. For the purpose of our study the temporal dimension in the impact function is ignored. Furthermore, both of our metrics express the impact at the global level and do not provide any information about local or regional impact. We emphasize that our metrics are simplified and chosen mainly for illustrative purposes and to provide a clean analysis and comparison. Section 2 describes the analysis, results are presented and discussed in Section 3 and conclusions are given in Section 4.

\section{Analysis}

Given the uncertainties in the climate response to imposed forcings, we present results using output from two GCMs (ECHAM4 coupled to a slab ocean and HadSM3 (Williams et al. 2001; Stenke et al. 2008)) in order to represent at least some inter-model difference. Equilibrium climate change GCM integrations were performed for global $\mathrm{CO}_{2}$ and $\mathrm{CH}_{4}$ increases and for perturbations of $\mathrm{O}_{3}$ from aviation, road traffic and shipping resulting from present-day emissions (Ponater et al. 2009). Concentration changes are calculated using chemical transport models. The HadSM3 also performed perturbations of black carbon (BC), organic carbon $(\mathrm{OC})$ and sulfate $\left(\mathrm{SO}_{4}\right)$ resulting from emissions from the transport sectors (Shine et al. 2011). Details of the simulations and data are given the supporting information (SI). 
In our analysis we use the surface air temperature (SAT) change, i.e. the difference between each of the perturbed simulations and the reference case, and apply two simplified indicators for the global impact caused by the change in temperature. The basis for our definition of metrics for climate impact is the impact (or damage) function $D=\alpha(\Delta T)^{n}$, which has been used in several economic studies (Kandlikar 1995, 1996; Manne et al. 1995; Hammitt et al. 1996). Here $\alpha$ is a factor converting impact to monetary terms and the dependence on temperature change is often taken to be linear, quadratic or cubic $(n=1,2,3)$. Any spatially integrated estimate has the disadvantage that it hides the distribution of impacts. Still, from reasons of applicability there is a need for synthesis and aggregation, and assessments are often made on the global-mean level, i.e. using global-mean temperature as input.

Following this we first assume that the global impact can be expressed as the square of the global-mean temperature change, $(\overline{\Delta T})^{2}$, where the overbar indicates a global average. We call this our standard metric. In the framework of this paper we ignore the factor $\alpha$. Next we define an alternative metric assuming that any temperature change causes an impact which varies as the square of the temperature change, that is $\overline{(\Delta T)^{2}}$. Thus, instead of using global-mean values as input, the impact is now calculated locally at each grid point and then averaged globally. This alternative metric is less dependent on any global cancellation that may occur for the standard metric and hence may retain important information about the true impact. If there is a non-linear relationship between surface temperature change and climate impact, the spatial variability in response can influence a global-mean metric. We therefore choose a quadratic relationship, $n=2$, in our metrics. In the case of $n=1$, the level at which we do the global averaging would make no difference. Another aspect of the choice of $n=2$ is that the impact of temperature change is symmetric about the no-perturbation case. We do not assert that this symmetry is, in fact, realistic. As emphasized in the introduction, our simplified impact functions are mainly serving illustrative purposes.

Our alternative metric corresponds to the mean square and the relationship between the two metrics applied here can be expressed as

$$
\Delta T_{M S}^{2}=(\overline{\Delta T})^{2}+\sigma^{2}
$$

which in turn can be written

$$
\frac{\sum \Delta T_{i}^{2}}{N}=\left(\frac{\sum \Delta T_{i}}{N}\right)^{2}+\sigma^{2}
$$

where $\Delta \mathrm{T}_{\mathrm{i}}$ is temperature change at point $\mathrm{i}, \mathrm{N}$ is the number of grid points and $\sigma^{2}$ gives the difference between the two approaches. The alternative metric has the characteristic that it increases with the degree of spatial heterogeneity. We use annual-mean temperature changes, averaged over all model years. This reduces the degree of spatial heterogeneity compared to using e.g. single monthly means and we thus ignore any seasonal or shorter term component.

We further define a heterogeneity ratio $(H R)$ as the ratio of the alternative and standard metric

$$
H R=\frac{\overline{\left(\Delta T^{2}\right)}}{(\overline{\Delta T})^{2}}
$$

A ratio higher than 1 shows that the response is heterogeneous and we capture more of the regional variability than when the standard metric is applied, thus obtaining a more complete signal in the calculation of global impacts. 
Even a homogeneous concentration change, such as an increase in $\mathrm{CO}_{2}$, causes an inhomogeneous forcing and a consequent temperature response with a distinct geographical distribution due to dynamics and spatial variability in the strength of feedbacks in the climate system. Boer and Yu (2003) investigated the relationship between radiative forcing (RF) and global-mean SAT response and found a tendency for a generic temperature response that depends mainly on the distribution of the climate feedbacks, and only secondarily on the forcing pattern. This view is supported by Lambert et al. (2011). In order to explore how important the patterns of climate feedbacks/dynamics and RF are, respectively, for the heterogeneity in the response to the transport perturbations, we define the normalized heterogeneity ratio (NHR)

$$
N H R_{i}=\frac{H R_{i}}{H R_{C O 2 \text { transport }}}
$$

for transport sector $\mathrm{i}$. This is the heterogeneity ratio for the short-lived perturbations in each transport sector normalized by that of $\mathrm{CO}_{2}$ from total transport (see $\mathrm{SI}$ ). (Because $\mathrm{CO}_{2}$ is so long-lived, the heterogeneity associated with transport $\mathrm{CO}_{2}$ is no different to the heterogeneity associated with other sources of $\mathrm{CO}_{2}$.) Since the same dynamics and feedbacks affect both the response to the $\mathrm{CO}_{2}$ perturbation and to the transport perturbations (this might not be exactly the case for indirect aerosol effects, however we only consider indirect effects in a simplified way - see SI), we are left with the signal caused by the departure of the pattern of forcing from the more homogeneous $\mathrm{CO}_{2}$ case. The ratio thus quantifies the excess of heterogeneity in the temperature change that can be directly attributed to the spatial distribution of the transport related non- $\mathrm{CO}_{2}$ forcings.

\section{Results and discussion}

\subsection{Climate impact of ozone precursor emissions}

Figure 1a to $\mathrm{f}$ shows the annual-mean SAT change due to ozone precursor emissions (OPEnet of $\mathrm{O}_{3}, \mathrm{CH}_{4}$ and primary mode $\mathrm{O}_{3}\left(\mathrm{O}_{3} \mathrm{PM}\right)$, see $\left.\mathrm{SI}\right)$ from aviation, road traffic and shipping with ECHAM4 and HadSM3. The temperature change from aviation and road traffic OPE shows a significant inter-hemispheric difference, with largest change in the Northern hemisphere where the majority of emissions occur. There is also a longitudinal pattern, which is similar for all sectors despite the different distributions of emissions and $\mathrm{RF}$, and which resembles the well-established land/ocean response for more homogeneous forcing such as $\mathrm{CO}_{2}$ (Fig. $1 \mathrm{~g}$ and $\mathrm{h}$ ). The land/sea contrast, i.e. temperature over land areas responding more strongly than over oceans, favors Northern hemisphere temperature change and has been demonstrated to be a robust feature of modelled transient and equilibrium climate change (e.g. Sutton et al. 2007). Studies suggest that for more uniform forcings the temperature response depends on local response and global-mean forcing, and less on the forcing pattern (Boer and Yu 2003; Lambert et al. 2011). However, Shindell et al. (2010) found that for forcings that are highly inhomogeneous the surface temperature response is fairly sensitive to the latitude of the forcing, but less so to the longitude. Though exhibiting significant geographical variability, the SAT change due to shipping OPE shows less interhemispheric difference than the two other sectors (because the $\mathrm{O}_{3}$ forcing itself has less inter-hemispheric difference - see Myhre et al. (2011) and because the more homogeneous $\mathrm{CH}_{4}$ and $\mathrm{O}_{3} \mathrm{PM}$ forcings dominate) and is, unlike the other sectors, negative everywhere. 
Fig. 1 Annual-mean surface air temperature change $\left[{ }^{\circ} \mathrm{C}\right]$ due to ozone precursor emissions (OPE). ECHAM4 model results are in the left hand column, HadSM3 in the right hand column. a and $\mathbf{b}$ aviation; $\mathbf{c}$ and $\mathbf{d}$ road traffic; e and $\mathbf{f}$ shipping. $\mathbf{g}$ and $\mathbf{h}$ show the change due to $\mathrm{CO}_{2}$ from the transport sector as a whole. Note the different scales

This is due to the strong negative RF of shipping $\mathrm{CH}_{4}$ and $\mathrm{O}_{3} \mathrm{PM}$ (Table SI1). The geographical distribution is also more similar to that of total transport $\mathrm{CO}_{2}$, with the largest temperature changes (though of opposite sign) occurring in high latitudes in both hemispheres.

The zonal- and annual-mean SAT change by component $\left(\mathrm{O}_{3}, \mathrm{CH}_{4}\right.$ and $\left.\mathrm{O}_{3} \mathrm{PM}\right)$ from each transport sector and model is shown in Fig. 2, together with the net temperature change. Changes in $\mathrm{O}_{3}$ cause warming at all latitudes and show the largest inter-hemispheric difference. $\mathrm{CH}_{4}$ causes a more symmetric cooling (as does $\mathrm{O}_{3} \mathrm{PM}$, but as noted in Section $\mathrm{SI} 1$, this response is assumed to be simply a scaled version of the $\mathrm{CH}_{4}$ response). The net zonal-mean temperature change is positive for road traffic OPE, while shipping OPE has a cooling impact. Aviation OPE causes a net warming in the Northern hemisphere, but a slight cooling in the Southern hemisphere in HadSM3, while the temperature change is positive at all latitudes in ECHAM4.

Next we aggregate the information to the global level and compare our two metrics for climate impact. The results of our analysis of the climate impact of total transport $\mathrm{CO}_{2}$ and OPE from the transport sectors are summarized in Table 1. The first column gives the globalmean temperature change calculated from the spatial distributions in Fig. 1. The net RF of $\mathrm{O}_{3}, \mathrm{CH}_{4}$ and $\mathrm{O}_{3} \mathrm{PM}$ is strongest for road traffic (Table SI1), resulting in the highest globalmean temperature change from this sector, while shipping causes a net cooling. For all sectors, the temperature change due to OPE is significantly smaller than that of total transport $\mathrm{CO}_{2}$.

Looking at the global climate impact given by our two metrics, road traffic is again the most important sector. However, because our metrics implicitly give equal weight to positive and negative temperature changes, shipping causes a larger impact than aviation. Applying the alternative metric does not change the ranking of sectors in terms of their impact compared to the standard calculation.

Our metrics, and thus the heterogeneity in the temperature change, can be compared in terms of absolute difference given by $\sigma^{2}$ (Eq. 1) or the heterogeneity ratio, HR (Eq. 3), given in Table 1. Looking at HR it is clear that of the three transport subsectors, aviation gives the most heterogeneous response: the impact is $\sim 90 \%$ higher for ECHAM4 when we apply the alternative metric compared to the standard metric. For road traffic and shipping the difference between the metrics is $27 \%$ and $14 \%$, respectively. The response in HadSM3 is generally more heterogeneous, especially for aviation OPE, where the alternative metric gives a 7 times higher climate impact than the standard metric.

Aviation OPE in HadSM3 shows the largest ratio of Northern to Southern hemisphere temperature change and net negative temperature change in the Southern hemisphere, which leads to strong cancellations in the global-mean. For HadSM3, 50\% of the additional signal in the alternative metric can be attributed to this inter-hemispheric difference in temperature response for aviation. This is demonstrated by calculating the metrics assuming that we have only two gridboxes (a Northern and Southern hemisphere, i.e. $N=2$ in Eq. 2). In this case the only heterogeneity contributing to the difference between metrics, given by HR, is the interhemispheric difference and we compare this with the HR calculated using all gridpoints to see how much of the additional signal that is retained in the $N=2$ case. Corresponding values are somewhat lower for road traffic and shipping in HadSM3 and in ECHAM4. We note that 
a)

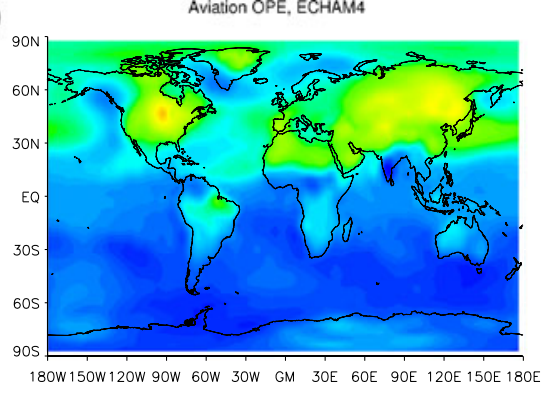

$\begin{array}{llllllll}-0.010 & -0.005 & 0.001 & 0.006 & 0.012 & 0.017 & 0.023 & 0.028\end{array}$

c)

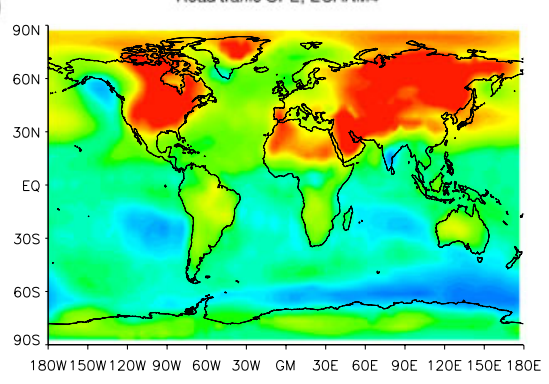

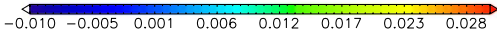

e)

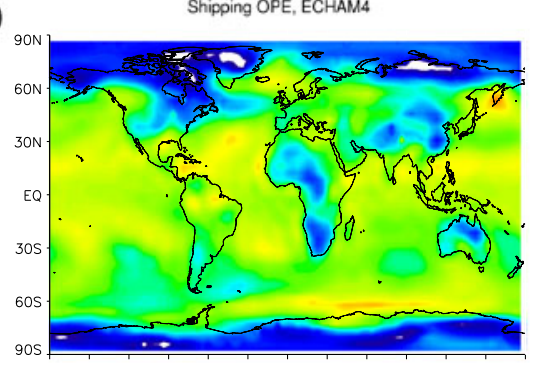

180W150W120W 90W 6OW 3OW GM 3OE GOE GOE 120E 150E $180 E$

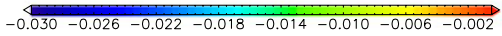

g)

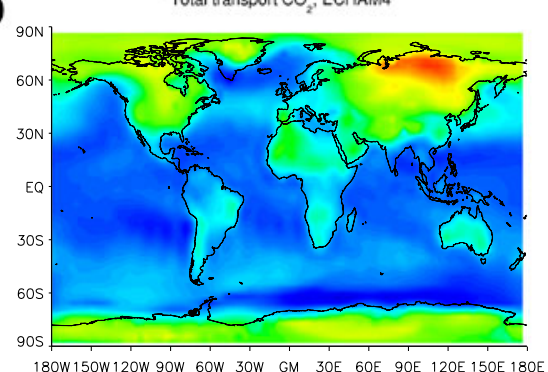

\begin{tabular}{lllllllll}
\hline 0.00 & 0.04 & 0.08 & 0.13 & 0.17 & 0.21 & 0.25 & 0.29 & 0.34
\end{tabular} b)
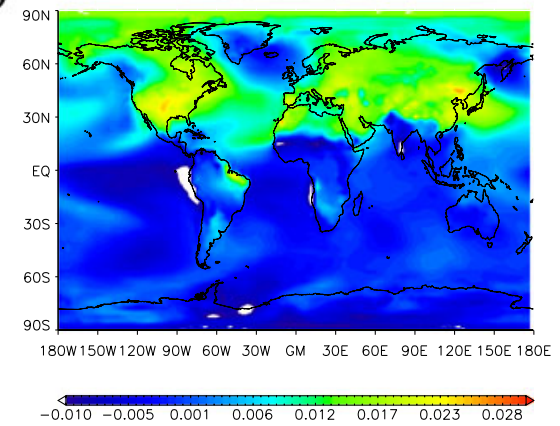

d)

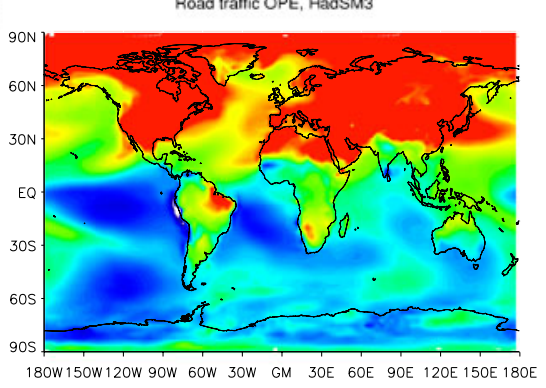

f)
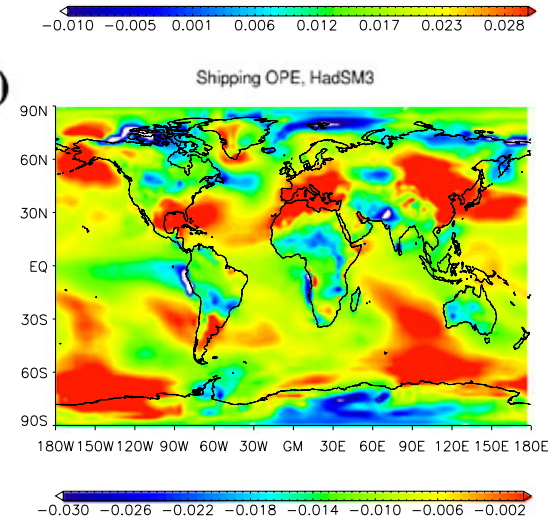

h)

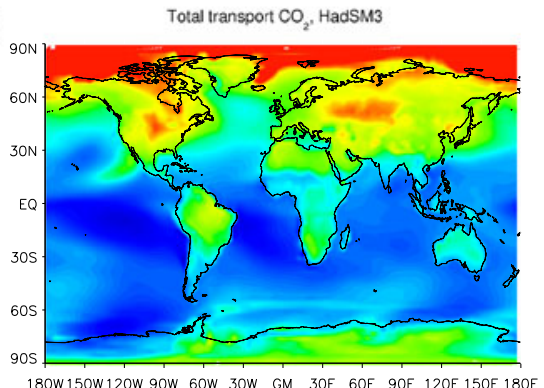

$0.000 \quad 0.041 \quad 0.0810 .1220 .1620 .203 \quad 0.2430 .2840 .325$ 

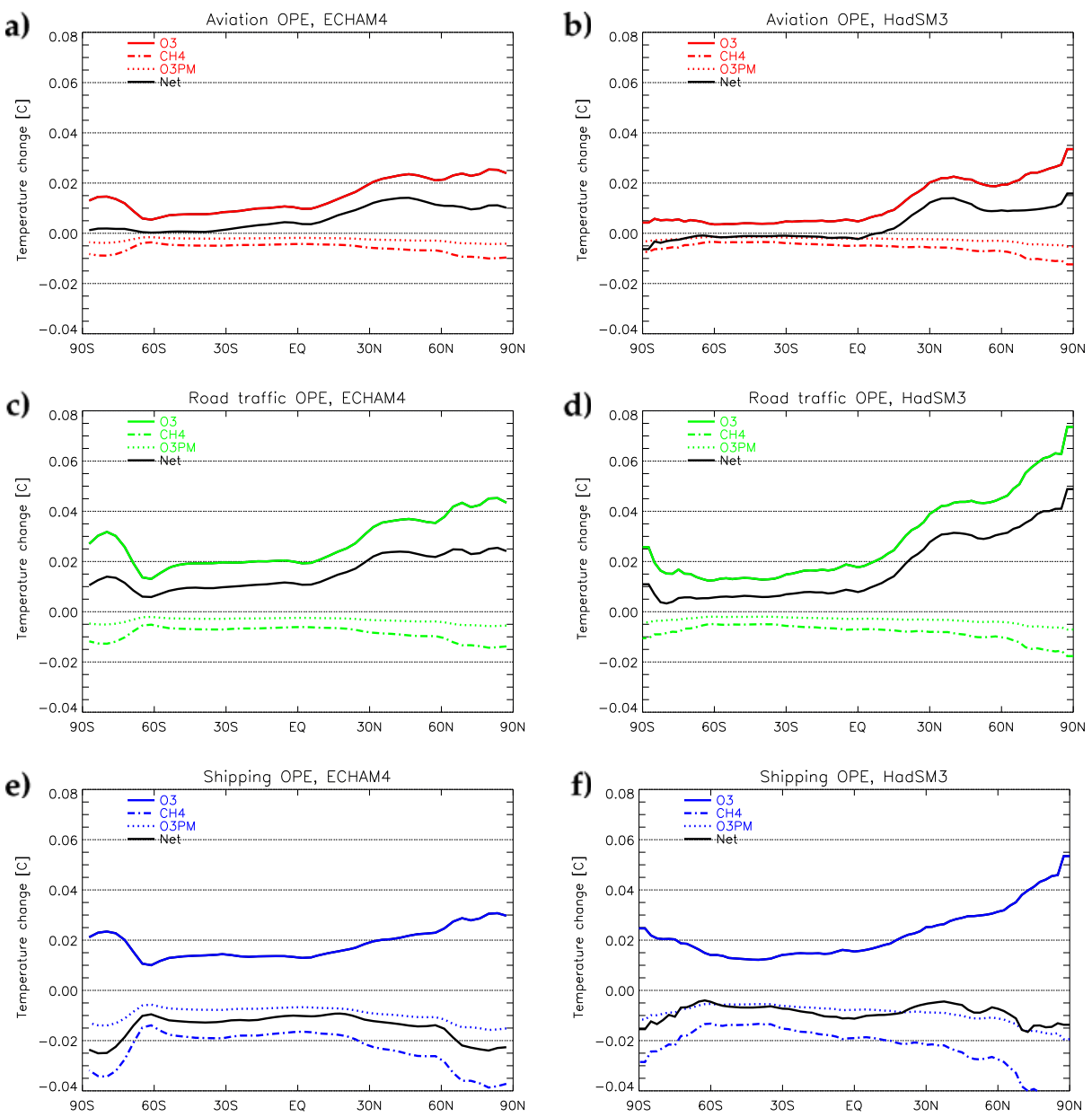

Fig. 2 Annual, zonal-mean surface air temperature change $\left[{ }^{\circ} \mathrm{C}\right]$ by component for $\mathrm{O}_{3}$ (solid), $\mathrm{CH}_{4}$ (dotdashed) and $\mathrm{O}_{3} \mathrm{PM}$ (dashed). ECHAM4 model results are in the left hand column, HadSM3 in the right hand column. a and $\mathbf{b}$ aviation; $\mathbf{c}$ and $\mathbf{d}$ road traffic; e and $\mathbf{f}$ shipping. The solid black line shows net temperature change of these components for each sector

because the metric values are very small, particularly for aviation, the HR values can get quite high. In terms of absolute values (i.e. $\sigma^{2}$ ) the difference between metrics is largest for road traffic. However, this sector has a larger climate impact due to higher emissions of ozone precursors. When normalized by emissions, the absolute difference between metrics is largest for aviation, consistent with HR. By applying the alternative metric we are able to detect a more complete signal of impact by capturing more of the spatial variability and we find that we are losing most information by standard global averaging in the case of aviation OPE.

Although perturbations of $\mathrm{CO}_{2}$ concentration give a more homogeneously distributed forcing and temperature response than perturbations of short-lived species, the response still shows a distinct geographical pattern (Fig. $1 \mathrm{~g}$ and h, Table 1). The normalized heterogeneity ratio, NHR (Eq. 4), shows the part of the additional signal in the alternative metric which is 
Table 1 Summary of standard and alternative metrics, heterogeneity ratios (HR) and normalized heterogeneity ratios (NHR) for $\mathrm{CO}_{2}$ from the transport sector as a whole and for ozone precursor emissions (OPE) from aviation, road traffic and shipping using results from ECHAM4 (top) and HadSM3 (bottom)

Global mean $\Delta \mathrm{T}\left[{ }^{\circ} \mathrm{C}\right] \quad$ Standard metric $\left[{ }^{\circ} \mathrm{C}\right]^{2} \quad$ Alternative metric $\left[{ }^{\circ} \mathrm{C}\right]^{2} \quad \mathrm{HR} \quad \mathrm{NHR}$

\begin{tabular}{llllll}
\hline ECHAM4 & & & & & \\
Total transport $\mathrm{CO}_{2}$ & 0.14 & 0.019 & 0.022 & 1.13 & 1 \\
Aviation OPE & 0.006 & $3.3 \mathrm{E}-05$ & $6.2 \mathrm{E}-05$ & 1.88 & 1.66 \\
Road traffic OPE & 0.014 & $2.1 \mathrm{E}-04$ & $2.6 \mathrm{E}-04$ & 1.27 & 1.12 \\
Shipping OPE & -0.012 & $1.6 \mathrm{E}-04$ & $1.8 \mathrm{E}-04$ & 1.14 & 1.01 \\
HadSM3 & & & & & \\
Total transport $\mathrm{CO}_{2}$ & 0.15 & 0.024 & 0.028 & 1.21 & 1 \\
Aviation OPE & 0.003 & $8.4 \mathrm{E}-06$ & $6.1 \mathrm{E}-05$ & 7.3 & 6.0 \\
Road traffic OPE & 0.015 & $2.3 \mathrm{E}-04$ & $3.9 \mathrm{E}-04$ & 1.69 & 1.40 \\
Shipping OPE & -0.008 & $6.8 \mathrm{E}-05$ & $1.1 \mathrm{E}-04$ & 1.56 & 1.30 \\
\hline
\end{tabular}

caused by the departure of the pattern of forcing from the more homogeneous $\mathrm{CO}_{2}$ case and indicates how important the spatial distribution of climate feedbacks and RF, respectively, are for the pattern of temperature response. A NHR higher than 1 means heterogeneity in forcing is more important than for the $\mathrm{CO}_{2}$ case. In our analysis NHR differs from 1 for road traffic and aviation OPE in both models, and for shipping in HadSM3. In the case of shipping in ECHAM4, however, the heterogeneity is similar to the heterogeneity for transport $\mathrm{CO}_{2}$, a result which was seen in Fig. 1 and is consistent with $\mathrm{CH}_{4}$ being the more dominant $\mathrm{RF}$ in this case versus the stronger $\mathrm{RF}$ of $\mathrm{O}_{3}$ for the other two transport sectors.

To further investigate the contributions of longitudinal and latitudinal variability in the temperature change (Fig. 1) to our analysis we repeat the calculations of metrics, but now for the (1D) zonally-averaged temperature fields (Table 2), and compare with the results from the analysis with the $2 \mathrm{D}$ temperature field above. Going over to zonally-averaged data cannot (by

Table 2 Summary of standard and alternative metrics, heterogeneity ratios (HR) and normalized heterogeneity ratios (NHR) for $\mathrm{CO}_{2}$ from the transport sector as a whole and for ozone precursor emissions (OPE) from aviation, road traffic and shipping using results from ECHAM4 (top) and HadSM3 (bottom). Values are calculated using zonal-mean surface temperature changes

Global mean $\Delta \mathrm{T}\left[{ }^{\circ} \mathrm{C}\right] \quad$ Standard metric $\left[{ }^{\circ} \mathrm{C}\right]^{2}$ Alternative metric $\left[{ }^{\circ} \mathrm{C}\right]^{2}$ HR NHR

\begin{tabular}{llllll}
\hline ECHAM4 & & & & & \\
Total transport $\mathrm{CO}_{2}$ & 0.14 & 0.019 & 0.020 & 1.07 & 1 \\
Aviation OPE & 0.006 & $3.3 \mathrm{E}-05$ & $5.4 \mathrm{E}-05$ & 1.63 & 1.53 \\
Road traffic OPE & 0.014 & $2.1 \mathrm{E}-04$ & $2.4 \mathrm{E}-04$ & 1.16 & 1.08 \\
Shipping OPE & -0.012 & $1.6 \mathrm{E}-04$ & $1.7 \mathrm{E}-04$ & 1.07 & 1.00 \\
HadSM3 & & & & & \\
Total transport $\mathrm{CO}_{2}$ & 0.15 & 0.024 & 0.027 & 1.14 & 1 \\
Aviation OPE & 0.003 & $8.4 \mathrm{E}-06$ & $4.1 \mathrm{E}-05$ & 4.9 & 4.3 \\
Road traffic OPE & 0.015 & $2.3 \mathrm{E}-04$ & $3.5 \mathrm{E}-04$ & 1.49 & 1.31 \\
Shipping OPE & -0.008 & $6.8 \mathrm{E}-05$ & $7.4 \mathrm{E}-05$ & 1.09 & 0.95 \\
\hline
\end{tabular}


our definitions) affect the standard metric, but the effect of longitudinal variability will not be captured in the alternative metric.

We use the heterogeneity compared to that of $\mathrm{CO}_{2}$ (i.e. NHR) for each transport sector and calculate the ratio of the $2 \mathrm{D}$ and $1 \mathrm{D}$ analyses:

$$
R=\frac{\left(N H R_{i}-1\right)_{2 D}}{\left(N H R_{i}-1\right)_{1 D}}
$$

where 1 is the NHR for $\mathrm{CO}_{2}$. In the case of aviation and road traffic OPE we find that we lose between $25 \%$ and $50 \%$ of the heterogeneity signal when using $1 \mathrm{D}$ instead of $2 \mathrm{D}$ data. This means that the stronger climate impact suggested by the alternative metric is largely caused by the fact that we capture more of the latitudinal heterogeneity, though the longitudinal pattern of temperature change also contributes to the increased signal. Our analysis shows that although the pattern of temperature response does not closely match the pattern of the forcing, the forcing pattern still influences the response pattern on a hemispheric scale. In particular, if a forcing is mostly in one hemisphere, then so is the response. This is supported by the findings in Shindell et al. (2010). In the case of shipping, the NHR is close to 1 for both models in the $1 \mathrm{D}$ analysis. The latitudinal variability in the response to shipping OPE is thus close to that of total transport $\mathrm{CO}_{2}$, and the more complete signal reflected by the higher values of the alternative metric is dominated by the longitudinal pattern, as is obvious from Fig. 1e and $\mathrm{f}$.

The climate sensitivity differs amongst the different forcing components (i.e. each component has its own characteristic climate efficacy (Hansen et al. 2005)) and this is implicitly included in our SAT changes. For a single forcing component or mechanism, the efficacy only describes the modification of the global-mean effect, not the pattern. However, for the net impact of a set of components, efficacy can contribute to the heterogeneity by affecting the relative importance of the response to individual components which determines the overall pattern. To investigate how this affects our analysis, we calculate the metrics and ratios assuming an efficacy of 1 , i.e. equal climate sensitivity parameters, for all perturbations. These results are then compared to the original calculations. For aviation and shipping we find somewhat lower heterogeneity ratio, while there is almost no effect on the results for road traffic. We thus conclude that the main signal in our alternative metric is due to differences in the heterogeneity in forcing and the consequent temperature response, rather than differences in efficacy.

\subsection{Climate impact of aerosols}

In addition to $\mathrm{O}_{3}$ and $\mathrm{CH}_{4}$, there are several other components from transport that have distinctly heterogeneous forcings, mainly $\mathrm{BC}$ and $\mathrm{SO}_{4}$, and aviation cirrus and contrails. In this section we extend our analysis to include also temperature change from $\mathrm{BC}, \mathrm{OC}$ and $\mathrm{SO}_{4}$ (with a simplifying assumption that patterns of surface temperature change are the same for the direct and first indirect (i.e. cloud albedo) forcings) from transport, using data from simulations with HadSM3 (Shine et al. 2011). Note that we have been selective in our inclusion of indirect effects - the cloud albedo forcing associated with $\mathrm{SO}_{4}$, whilst uncertain, is arguably the least uncertain of any of the cloud indirect effects; at the other extreme, the impact of aviation BC emissions on cirrus clouds, is highly uncertain, to the extent that not even the sign is known (Lee et al. 2010). The HadSM3 simulations implicitly include a representation of any semi-direct effects of $\mathrm{BC}$ on clouds (via changes in local relative humidity and stability) although these effects are likely to be strongly model dependent. For 
aviation, the direct aerosol effect is believed to be negligible (Table SI1) and so we focus only on road traffic and shipping.

For road traffic, zonal-mean temperature is similar in the Southern hemisphere in both the OPE case and in the case of combined ozone precursors and aerosols (OPE+AER), while there is an increased warming in the Northern hemisphere due to the addition of BC (Fig. SI1). Because of the strong negative $\mathrm{RF}$ of $\mathrm{SO}_{4}$, shipping causes a stronger cooling in the $\mathrm{OPE}+\mathrm{AER}$ case. The difference between our metrics is more pronounced for the OPE+AER road traffic case (Table 3) than for OPE alone (Table 1). The opposite is the case for shipping.

\subsection{Transport perturbations superimposed on a background atmosphere}

When perturbations are compared to some reference climate change, it is not evident that a negative temperature change leads to an overall cooling of climate-it could in fact only act to reduce an already existing warming trend. Furthermore, in the analysis above both positive and negative temperature changes are given equal significance through our assumption of a quadratic relationship between temperature change and impact. However, it is not obvious that a reduction in temperature would cause the same impact, or damage, as an increase. To explore the consequences of assuming that the transport forcing is superimposed on other forcings, we combine the temperature change due to OPE for each sector with the equilibrium temperature change representative of total anthropogenic $\mathrm{CO}_{2}$ since pre-industrial times to calculate the total impact $\left(\mathrm{D}_{\text {total }}\right)$. We thus assume that the forcing from $\mathrm{CO}_{2}$ is an approximation to the total anthropogenic forcing (which Forster et al. (2007) show may indeed be the case for the present-day, although the net forcing is much more uncertain) and look at the effect of the transport perturbations put on top. We are now looking at the contribution of OPE from current transport to the total present-day impact of human activity, $\Delta \mathrm{D}=\mathrm{D}_{\text {total }}-\mathrm{D}_{\mathrm{CO} 2}$.

While we expect larger heterogeneity due to larger inter-hemispheric difference and nonlinearity, we now find smaller heterogeneity ratios (Table SI2) than in the isolated OPE case (Table 1). We thus lose less information about regional variability when comparing the impact of short-lived species to the reference climate change. Meanwhile, since we now have a situation with higher background temperature, the climate impact in terms of our two metrics from the transport sectors is larger than in the isolated OPE case. One explanation for the lower heterogeneity we now observe is that the response pattern due to $\mathrm{CO}_{2}$ now dominates and that the transport perturbations are not large enough to induce a substantial modification. This is supported by the fact that the heterogeneity in the transport temperature

Table 3 Summary of standard and alternative metrics, heterogeneity ratios and normalized heterogeneity ratios for $\mathrm{CO}_{2}$ from the transport sector as a whole and for the combined temperature change due to ozone precursor emissions and aerosols $\left(\mathrm{BC}, \mathrm{OC}, \mathrm{SO}_{4}\right.$ direct and indirect) (OPE+AER) from road traffic and shipping using results from HadSM3

\begin{tabular}{llllll}
\hline & $\begin{array}{l}\text { Global mean } \Delta \mathrm{T} \\
{\left[{ }^{\circ} \mathrm{C}\right]}\end{array}$ & $\begin{array}{l}\text { Standard metric } \\
{\left[{ }^{\circ} \mathrm{C}\right]^{2}}\end{array}$ & $\begin{array}{l}\text { Alternative metric } \\
{\left[{ }^{\circ} \mathrm{C}\right]^{2}}\end{array}$ & HR & NHR \\
\hline HadSM3 & & & & & \\
Total transport $\mathrm{CO}_{2}$ & 0.15 & 0.024 & 0.028 & 1.21 & 1 \\
Road traffic OPE+AER & 0.04 & $1.4 \mathrm{E}-03$ & $2.6 \mathrm{E}-03$ & 1.86 & 1.54 \\
Shipping OPE+AER & -0.05 & $2.9 \mathrm{E}-03$ & $3.2 \mathrm{E}-03$ & 1.11 & 0.92 \\
\hline
\end{tabular}


responses, at least for ECHAM4, is not very different from $\mathrm{CO}_{2}$ (last column in Table SI2). To investigate the sensitivity to the magnitude of perturbations more closely, we perform several tests: (i) Temperature response due to $\mathrm{CO}_{2}$ doubling reduced by $50 \%$, (ii) temperature response to the transport sectors increased by a factor of 10 and (iii) both these changes simultaneously. All these changes have relatively little impact on the heterogeneity ratios.

The approach described in Sections 3.1 and 3.2 is in line with standard metrics such as GWP, which are applied on a constant $\mathrm{CO}_{2}$ background. Reisinger et al. (2011) found that changing background concentrations leads to notable changes in GWPs. We find that our metrics are quite sensitive to the background impact, i.e. the impact of the short-lived species depends on the background temperature change. The above results indicate that when we look at the transport sectors in isolation or compare them internally, global averaging can lead to significant loss of information about the impact, and accounting for the regional variability in global metrics can be important. However, when we assess the climate impact of small sectors or perturbations in connection with the total impact this becomes less important because the heterogeneity in the response to $\mathrm{CO}_{2}$ dominates. Using global-mean metrics may be justified in this case.

\subsection{Caveats}

While all our analyses show the same main conclusions and characteristics, the exact values of the results are quite sensitive to the magnitude of the perturbations and also to which components we include in the definition of net climate impact. For road traffic, the BC perturbation was performed with three different scaling factors: 250, 500 and 1,000. We perform the analysis using these different scalings and find that the difference between our standard and alternative metric is largest for the smallest perturbation. The temperature response to the BC perturbations is discussed in more detail in Shine et al. (2011), where it is shown that the inter-hemispheric difference is largest for the smaller scaling factors, perhaps due to a non-linearity in the semi-direct response of clouds to $\mathrm{BC}$. We also examine transport $\mathrm{O}_{3}$ perturbations scaled by two different factors (100 and 500), and find a much smaller impact on the analysis.

GCMs simulate a certain level of unforced variability that may mask the response induced by perturbations. If there are large numbers of insignificant grid-point responses, the additional signal we detect with the alternative metric could be affected by spurious spatial structures representing this unforced variability. To exclude this possibility, grid-point t-tests are performed for the response to ECHAM4 ozone perturbations. For the temporally averaged response to $\mathrm{O}_{3}$ from the transport sectors, the temperature signal is statistically significant at the $1 \%$ level in more than $99 \%$ of the grid-points.

A formal uncertainty analysis to determine the robustness of our normalized heterogeneity ratios and whether these are significantly different from 1 would require ensemble results. We do, however, perform a simplified uncertainty analysis by splitting our existing model runs into sub-means averaged over shorter time periods, thus obtaining a set of metric and ratio values (see SI). Results indicate that our analysis is robust and shows that the normalized heterogeneity ratios for OPE are significantly different from 1 .

We implicitly assume that the temperature change due to changes in $\mathrm{O}_{3}$ and $\mathrm{CH}_{4}$ can be added linearly to obtain the net response. Results from additional sensitivity simulations with ECHAM4 comparing the zonal and global-mean RF and temperature change for a combined $\mathrm{O}_{3}$ aviation and $\mathrm{CH}_{4}$ perturbation with the sum of these two perturbations suggests that this assumption is supported by the model results (see SI). Finally, uncertainty is related to the assumption that the forcing and response to $\mathrm{O}_{3} \mathrm{PM}$ and transport $\mathrm{CH}_{4}$ 
follows the pattern of the global $\mathrm{CH}_{4}$. This is also the case for the assumption that $\mathrm{SO}_{4}$ direct and indirect effects have the same distribution.

\section{Conclusions}

Using results from equilibrium climate change simulations with the ECHAM4 and HadSM3 GCMs, we have explored the heterogeneity in the surface air temperature response to ozone precursor emissions (including changes in ozone concentration derived from CTM calculations and the corresponding methane concentration changes) and aerosol emissions from road traffic, aviation and shipping. Ozone precursors are important components of the emissions from transport, but their net climate impact via changes in atmospheric ozone and methane is difficult to determine due to inhomogeneities in forcing and response, and compensational effects. Furthermore, both ozone precursors and aerosols produce more spatially heterogeneous RF than long-lived species like $\mathrm{CO}_{2}$ and the response can also be more heterogeneous. Using two simplified metrics for climate impact we investigate the loss of information about regional variability that results from the use of global-mean input in the case of heterogeneous temperature change and explore an alternative metric for capturing regional impacts. Though the possible loss of information about the distribution of impacts by global averaging is conceptually obvious, there is nevertheless widespread use of globalmean indicators and metrics. Our study takes a step towards quantifying the implications of this loss of information for specific sectors and components.

Significant longitudinal and latitudinal variability in the surface air temperature response to the perturbations in ozone, methane and aerosols from the transport sectors leads to global cancellations and loss of information when the climate impact is calculated using a metric based on input of global-mean temperature change. By applying a global metric which is calculated locally and then averaged globally, we capture more of the regional variability and detect a more complete signal of climate impact. This is most significant in the case of aviation, where the climate impact is a factor 2 and 7 larger for the ECHAM4 and HadSM3 respectively, when we account for regional variability in the calculation. An investigation of the contribution of longitudinal and latitudinal variability to the additional signal detected indicates that, although the pattern of temperature response does not closely match the pattern of the forcing, the forcing pattern still influences the response pattern in the case of short-lived components, at least on a hemispheric scale.

Standard metrics such as GWP are applied on a constant $\mathrm{CO}_{2}$ background. We superimpose the short-lived transport forcing on present-day anthropogenic $\mathrm{CO}_{2}$ forcing and find that our metrics are quite sensitive to the background impact. In this case the heterogeneity in the temperature response to $\mathrm{CO}_{2}$ dominates, which suggests that the importance of including regional climate impacts in global metrics depends on the type of analysis. If we consider the transport sectors in isolation or compare them among each other, global averaging can lead to significant loss of information about impact and accounting for regional variability in global metrics can be important. However, when we assess the climate impact of smaller sectors or perturbations in connection with an existing strong background impact, the difference between the two metrics becomes smaller and using global-mean metrics may be justified.

We use a relationship between climate change and impact which is often applied in literature and which would apply to any non-linear case. However, the exact relationship can in some cases be very uncertain. In other cases the relationship for a particular impact category may be known, but is strongly dependent on the impact of interest. Furthermore, 
we use annual-mean temperature responses averaged over all model years and consider only temperature change as input variable. Using results from two GCMs captures some of the inter-model variability and we provide a simplified uncertainty calculation to investigate the statistical robustness of our metrics.

Our analysis is a step towards quantifying the loss of small-scale spatial information about climate impacts and is valid when constructing metrics for application at the global scale. However, at this the stage the approach is not directly applicable to evaluating the change in impact due to specific emission changes, for instance by multiplying emissions with a metric to place them on a (for example) common "carbon-equivalent" scale, as is the case with the global warming potential (GWP). This is because the GCM simulations use prescribed concentration patterns derived from emission changes in CTM runs. The concentration changes can be sensitive to the location and seasonal (and perhaps even diurnal) timing of emissions. For a pulse emission of e.g. NOx the spatial heterogeneity would also be time dependent due to the different time scales of the impacts of $\mathrm{NOx}$ on $\mathrm{O}_{3}$ and $\mathrm{CH}_{4}$. For application in policy-making, this sensitivity needs to be accounted for. A possible future application of our approach is the inclusion of a measure of the spatial heterogeneity in the impact in metrics such as the GWP or global temperature change potential (GTP) (e.g. Fuglestvedt et al. 2010).

Acknowledgements This research was supported by the European Union's Sixth Framework Integrated Project QUANTIFY Contract No 003893 and the TEMPO project funded by the Norwegian Research Council. We thank Nicola Stuber for making available the results of the HadSM3 simulations.

\section{References}

Berntsen T, Fuglestvedt J (2008) Global temperature responses to current emissions from the transport sectors. Proc Natl Acad Sci U S A 105(49):19154-19159

Berntsen TK, Fuglestvedt JS et al (2005) Response of climate to regional emissions of ozone precursors: sensitivities and warming potentials. Tellus Ser B Chem Phys Meteorol 57(4):283-304

Boer GJ, Yu B (2003) Climate sensitivity and response. Clim Dyn 20(4):415-429

Forster PM, Blackburn M et al (2000) An examination of climate sensitivity for idealised climate change experiments in an intermediate general circulation model. Clim Dyn 16(10-11):833-849

Forster P, Ramaswamy V et al (2007) Changes in atmospheric constituents and in radiative forcing. Climate Change 2007: the physical science basis. Contribution of Working Group I to the Fourth Assessment Report of the Intergovernmental Panel on Climate Change. S. Solomon, D. Qin, M. Manninget al. Cambridge and New York, Cambridge Univ. Press

Fuglestvedt JS, Berntsen TK et al (1999) Climatic forcing of nitrogen oxides through changes in tropospheric ozone and methane; global 3D model studies. Atmos Environ 33(6):961-977

Fuglestvedt JS, Berntsen TK et al (2003) Metrics of climate change: assessing radiative forcing and emission indices. Clim Chang 58(3):267-331

Fuglestvedt J, Berntsen T et al (2008) Climate forcing from the transport sectors. Proc Natl Acad Sci U S A $105(2): 454-458$

Fuglestvedt JS, Shine KP et al (2010) Transport impacts on atmosphere and climate: metrics. Atmos Environ 44(37):4648-4677

Hammitt JK, Jain AK et al (1996) A welfare-based index for assessing environmental effects of greenhousegas emissions. Nature 381(6580):301-303

Hansen J, Sato M et al (2005) Efficacy of climate forcings. J Geophys Res Atmos 110(D18):D18104

IPCC (2009) Meeting Report of the Expert Meeting on the Science of Alternative Metrics. In: Plattner G-K, Stocker TF, Midgley P, Tignor M (eds). IPCC Working Group I Technical Support Unit, University of Bern, Bern, Switzerland, p 75

Joshi M, Shine K et al (2003) A comparison of climate response to different radiative forcings in three general circulation models: towards an improved metric of climate change. Clim Dyn 20(7-8):843854 
Joshi M, Hawkins E et al (2011) Projections of when temperature change will exceed 2 [deg]C above preindustrial levels. Nat Clim Chang 1(8):407-412

Kandlikar M (1995) The relative role of trace gas emissions in greenhouse abatement policies. Energy Policy 23(10):879-883

Kandlikar M (1996) Indices for comparing greenhouse gas emissions: integrating science and economics. Energy Econ 18(4):265-281

Lambert FH, Webb MJ et al (2011) The relationship between land-ocean surface temperature contrast and radiative forcing. J Clim 24(13):3239-3256

Lee DS, Pitari G et al (2010) Transport impacts on atmosphere and climate: aviation. Atmos Environ 44 (37):4678-4734

Manne A, Mendelsohn R et al (1995) A model for evaluating regional and global effects of GHG reduction policies. Energy Policy 23(1):17-34

Myhre G, Shine KP et al (2011) Radiative forcing due to changes in ozone and methane caused by the transport sector. Atmos Environ 45(2):387-394

Nordhaus WD, Boyer J (2000) Warming the world: economic models for global warming. MIT Press, Cambridge

Ponater M, Dietmüller S et al (2009) Indications of distinctive efficacies for transport related ozone perturbations. Second International Conference on Transport, Atmosphere and Climate (TAC-2), June 22-25 2009, Aachen and Maastricht

Reisinger A, Meinshausen M et al (2011) Future changes in global warming potentials under representative concentration pathways. Environ Res Lett 6(2):024020

Rive N, Torvanger A et al (2007) To what extent can a long-term temperature target guide near-term climate change commitments? Clim Chang 82(3-4):373-391

Shindell D, Faluvegi G (2010) The net climate impact of coal-fired power plant emissions. Atmos Chem Phys 10(7):3247-3260

Shindell D, Schulz M et al (2010) Spatial scales of climate response to inhomogeneous radiative forcing. J Geophys Res 115(D19):D19110

Shine KP, Berntsen TK et al (2005) Scientific issues in the design of metrics for inclusion of oxides of nitrogen in global climate agreements. Proc Natl Acad Sci U S A 102(44):15768-15773

Shine KP, Highwood EJ et al (2011) Climate model calculations of the impact of aerosols from road transport and shipping. In prep. for Atmospheric and Oceanic Optics

Skeie RB, Fuglestvedt J et al (2009) Global temperature change from the transport sectors: historical development and future scenarios. Atmos Environ 43(39):6260-6270

Stenke A, Grewe V et al (2008) Lagrangian transport of water vapor and cloud water in the ECHAM4 GCM and its impact on the cold bias. Clim Dyn 31(5):491-506

Sutton RT, Dong BW et al (2007) Land/sea warming ratio in response to climate change: IPCC AR4 model results and comparison with observations. Geophys Res Lett 34(2):L02701

Tol RSJ (2002) Estimates of the damage costs of climate change. Part II. Dynamic estimates. Environ Resour Econ 21(2):135-160

Tol RSJ, Downing TE et al (2004) Distributional aspects of climate change impacts. Glob Environ ChangeHum Policy Dimens 14(3):259-272

Unger N, Shindell DT et al (2008) Air pollution radiative forcing from specific emissions sectors at 2030. J Geophys Res Atmos 113(D2):D02306

Unger N, Shindell DT et al (2009) Climate forcing by the on-road transportation and power generation sectors. Atmos Environ 43(19):3077-3085

Williams KD, Senior CA et al (2001) Transient climate change in the Hadley Centre models: the role of physical processes. J Clim 14(12):2659-2674 